\title{
ANÁLISIS DE LA COLECCIÓN DE MURCIÉLAGOS (MAMMALIA: CHIROPTERA) DEL MUSEO NACIONAL DE HISTORIA NATURAL DE SANTO DOMINGO
}

\author{
Miguel S. Núñez Novas ${ }^{1}$ y Yolanda M. León ${ }^{2}$
}

\begin{abstract}
${ }^{1}$ Museo Nacional de Historia Natural de Santo Domingo (MNHNSD). Calle César Nicolás Penson, Plaza de la Cultura, Santo Domingo, República Dominicana.m.nunez@museohistorianatural.gov.do ${ }^{2}$ Instituto Tecnológico de Santo Domingo y Grupo Jaragua, Santo Domingo, República Dominicana. ymleon@intec.edu.do
\end{abstract}

\section{RESUMEN}

Se hace la descripción de la colección de murciélagos del Museo Nacional de Historia Natural de Santo Domingo. Se atiende a la composición de la colección y la distribución geográfica de sus especímenes; se hace una actualización taxonómica. Se examinaron 723 especímenes, pertenecientes a seis (6) familias, 17 géneros y 18 especies, estando representadas todas las especies reportadas para La Hispaniola. Todos los géneros son monoespecíficos, con excepción de Pteronotus Gray, 1838 con dos especies. Las fechas de colecta datan desde 1961 y se extienden hasta el 2008, correspondiendo la mayor parte de registros a la Región Suroeste de la República Dominicana. Las especies más abundantes en la colección resultaron ser generalistas en cuanto a selección de refugios, tales como los filostómidos Artibeus jamaicensis y Macrotus waterhousii y el molósido Molossus molossus. Cabe destacar la presencia de especies de cuevas calientes (Pteronotus parnellii, P. quadridens, y Phyllonycteris poeyi), así como especies raras: Chilonatalus micropus, Lasiurus minor y Natalus major.

Palabras clave: museo, colección, murciélagos, Chiroptera, La Hispaniola.

\section{ABSTRACT}

The collection of bats of the National Museum of Natural History of Santo Domingo is described focusing on its composition and the geographic distribution of its specimens. The opportunity is taken to update its taxonomic information. A total of 723 specimens belonging to 6 families, 17 genera and 18 species, representing all of the species reported for Hispaniola, were examined. All genera but Pteronotus Gray, 1838 with two species, are monospecific. Collection dates were from 1961 to 2008, with most of the records corresponding to the southwest of the Dominican Republic. Most species were generalists for site selection, such as the phyllostomids Artibeus jamaicensis and Macrotus waterhousii as well as the molossid Molossus molossus. The presence of hot-cave species (Pteronotus parnellii, P. quadridens, and Phyllonycteris poeyi) and the rare species (Chilonatalus micropus, Lasiurus minor and Natalus major) is reported.

Key words: museum, collection, bats, Chiroptera, Hispaniola.

\section{INTRODUCCIÓN}

Los murciélagos (Orden Chiroptera Blumenbach, 1779) constituyen el segundo grupo de mamíferos más diverso en el mundo, sólo superado por los roedores y comprendiendo más de 1,116 especies descritas, distribuidas en 18 familias (Wilson y Reeder, 2005); de estas familias, nueve (9) están presentes en América (Miller y Miller, 2002). En La Hispaniola, los murciélagos constituyen el 90\% de los mamíferos descritos hasta el momento, distribuidos en 6 familias y 18 especies. 
Los estudios que involucran este Orden, realizados exclusivamente en La Hispaniola, son limitados. Entre los trabajos publicados podemos mencionar la descripción de especies nuevas procedentes de la isla por Elliot (1905) y Miller (1918), la descripción de la colección de murciélagos de la isla de A. H. Verrill obtenida por el American Museum of Natural History, New York (Allen, 1908); el primer reporte de Noctilio leporinus (Armstrong y Johnson, 1969); el estudio de murciélagos del sur de Haití (Klingener et al., 1978); reportes sobre los murciélagos del Lago Enriquillo y la Isla Beata (Ottenwalder, 1978, 1979, 1981); los registros de especies de cuevas calientes (Tejedor et al., 2005a) y el reporte de mamíferos extintos en cuevas por McFarlane et al (2000).

Sin embargo, datos y/o muestras de los murciélagos de La Hispaniola han sido incluidos en varios estudios y análisis regionales, como el catálogo de mamíferos vivientes y extintos de las Antillas (Varona, 1974), varios trabajos sobre la zoogeografía de murciélagos antillanos (Baker y Genoways, 1978; Koopman, 1989; Rodríguez-Durán y Kunz, 2001), patrones de biodiversidad de quirópteros en las Antillas (Fleming, 1982; McFarlane, 1989 y 1991) y análisis de la distribución de murciélagos en las Antillas (Griffiths y Klingener, 1988). Además, la revisión del género Natalus de las Antillas (Tejedor et al., 2005b), filogenética molecular de la familia Natalidae (Dávalos, 2005) y descripción de las subespecies de Erophylla sezerkoni en el Caribe (Baker et al., 1978), así como la morfología de cráneos y la dieta de especies antillanas (Rodríguez-Durán et al., 1993).

Otra fuente importante de información sobre los murciélagos de La Hispaniola proviene de los especímenes preservados en las colecciones de instituciones internacionales, especialmente de Norteamérica. Entre estas se destaca la colección del Slater Museum of Natural History, University of Puget Sound (Washington, D.C., EEUU), seguido, en orden de cantidad de especímenes, por University of Kansas Biodiversity Research Center (Kansas, EEUU), Museum of Comparative Zoology, Harvard University (Massachusetts, EEUU), National Museum of Natural History, Smithsonian Institution (Washington, D.C., EEUU), University of California, Museum of Vertebrate Zoology, Berkeley, California (California, EEUU) y el Royal Ontario Museum (Ontario, Canadá), entre otros.

La colección de murciélagos del Museo Nacional de Historia Natural de Santo Domingo (MNHNSD) fue iniciada por José A. Ottenwalder a principios de la década del 1970, siendo él pionero en el estudio de estos mamíferos en La República Dominicana. Otros investigadores nacionales y extranjeros han hecho importantes contribuciones a la colección, tales como Don W. Stone, Nelson García Marcano, Julio Cicero, Sixto J. Incháustegui y Leonardo Salazar. Se obtuvieron donaciones de especímenes provenientes de colecciones privadas, en base a colectas realizadas en la década del 1960 por F. H. Arnmstrong, del Slater Museum of Natural History, University of Puget Sound. A partir del año 2006, la colección de murciélagos del MNHNSD empieza a formar parte de la colección mastozoológica del recién creado Departamento de Investigación y Conservación. Como parte de las labores iniciales de este Departamento, se realizó un inventario detallado de dicha colección, haciendo las debidas actualizaciones nomenclaturales.

A continuación presentamos el primer listado de la colección, así como varios análisis sobre su contenido y distribución en el territorio nacional. Esperamos que esto sirva de útil referencia en los estudios y trabajos de conservación de este diverso grupo de mamíferos en la isla. 


\section{OBJETIVOS}

- Hacer la actualización taxonómica del registro de la colección de murciélagos del MNHNSD.

- Realizar la curación de los especímenes preservados.

- Actualizar los datos geográficos de las colectas y, en los casos posibles, introducir las coordenadas aproximadas en un sistema de información geográfica que permita su análisis y la producción de mapas temáticos.

- Analizar la composición cuantitativa y cualitativa de la colección, así como la distribución geográfica de sus especímenes.

\section{MATERIALES Y MÉTODOS}

Se procedió a revisar los especímenes de murciélagos preservados y depositados en el MNHNSD hasta el 30 de septiembre de 2009. La información de cada espécimen registrada en su etiqueta fue verificada en el catálogo de la colección mastozoológica preparado por J. A. Ottenwalder. Los nombres de localidades que poseían las colectas fueron actualizados utilizando la más reciente división territorial de La República Dominicana (ONE, 2006). Sin embargo, algunas localidades de colecta no poseían suficiente detalle para poder ser completadas. Las localidades ubicadas o, al menos, el poblado más cercano con el nombre de la localidad, fueron incorporadas mediante sus coordenadas aproximadas a un sistema de información geográfica con el programa ArcGIS, versión 8.3 (ESRI, California), para su análisis y producción de mapas temáticos. En todos los casos, la información original de la colecta se conservó en la etiqueta vieja junto a los especímenes correspondientes.

Los ejemplares se encontraron preservados en tres formas: alcohol, osamenta y piel. Algunos especímenes presentaron más de una forma de preservación (por ejemplo, cráneo en seco y cuerpo en húmedo, etc.; fig. 1). A estos se les dio el mantenimiento necesario: cambio de alcohol, eliminación de hongos del material en seco, restitución de etiquetas y cambio de frascos en mal estado, entre otras acciones.

Actualización taxonómica. Se realizó una actualización taxonómica, de acuerdo a los cambios nomenclaturales registrados. Al mismo tiempo, se determinó la especie para los especímenes que aparecieron sin identificación. En ninguno de los casos se reemplazaron etiquetas ni se alteró la información aparecida en el catálogo numérico; en los casos necesarios la nueva información se registró en una nueva etiqueta que se colocó con su nueva fecha junto a la ya existente. Los cambios se registraron en los formularios de un nuevo catálogo taxonómico complementario del catálogo numérico. Las actualizaciones se hicieron de acuerdo a: Anderson (1969); Homan y Jones (1975); Baker et al (1978, 1984); Silva (1979); Hoyt y Baker (1980); Shump y Shump (1982); Herd (1983); Swanepoel y Genoways (1983); Hood y Jones (1984); Wilkins (1989); Kurta y Baker (1990); Milner et al (1990); Rodríguez-Durán y Kunz (1992); Lancaster y Kalko (1996); Pierson y Rainey (1998); Ortega y Castro-Arellano (2001) y Timm y Genoways (2003). La actualización realizada consideró el problema de las sinonimias existentes, con la consiguiente unificación de los nombres en los casos pertinentes. A estos fines se siguió a Simmons (2005), exceptuando los casos de Brachyphylla pumila y Nyctinomops macrotis, para los cuales se siguió a Murray (2008) y Pierson y Rainey (Ídem), respectivamente. 


\section{RESULTADOS Y DISCUSIÓN}

Composición. Se examinó un total de 723 especímenes, distribuidos en 6 familias, 17 géneros y 18 especies. La colección tiene representación de todas las especies registradas para la isla. Cabe destacar que todos los géneros en la colección (y la isla) son monoespecíficos con la excepción de Pteronotus, que comprende dos especies: P. parnellii y P. quadridens (fig. 2).

De las seis familias registradas, Phyllostomidae es la que posee la mayor cantidad de especies (7), tanto registradas para la isla como presentes en la colección. Igualmente, resultó la familia con mayor número de especímenes, dominando la especie Artibeus jamaicensis $(20 \%$ de los especímenes). Otro filostómido, Macrotus waterhousii, ocupa el segundo lugar (14\%). Sigue en abundancia la familia Molossidae, con la especie Molossus molossus como dominante (12\%). Las especies más pobremente representadas en la colección son Chilonatalus micropus, con tres (3) especímenes y Natalus major, con siete (7) especímenes (familia Natalidae), así como Lasiurus minor con cuatro (4), perteneciente a la familia Vespertilionidae.

La dominancia de $A$. jamaicensis en la colección no fue sorprendente, pues se conoce que es un murciélago que puede utilizar una amplia gama de hábitats y refugios, como cuevas, árboles y edificaciones, entre otros (Silva, 1979; Ortega y Castro-Arellano, 2001). La segunda especie en cantidad de especímenes, Macrotus waterhousii, es un murciélago insectívoro que puede habitar cavernas abiertas (Silva, 1979), lo cual podría facilitar su detección y colecta.

La abundancia de $M$. molossus en la colección igualmente pudiera explicarse por su conocida adaptación a los ambientes antrópicos (Silva, 1979). Sampedro-Marín et al (2008) consideran que $M$. molossus posee preferencia por las construcciones humanas, tanto habitadas como no habitadas, seguramente por las mayores posibilidades que les brindan estos refugios y por la cercanía con sus fuentes principales de alimento, los insectos, que suelen concentrarse en algunos lugares de pueblos y ciudades, dada la iluminación en estos sitios.

Es importante notar la presencia de murciélagos asociados a "cuevas calientes", tales como P. parnellii, P. quadridens y Phyllonycteris poeyi (Rodríguez-Durán y Kunz, 1992; Silva, 1979; Herd, 1983). Las cuevas calientes se caracterizan por presentar temperaturas entre 26 y $40^{\circ} \mathrm{C}$ y una humedad relativa superior a 90\% (Silva, 1977; Rodríguez-Durán, 1998). Este tipo de cuevas ha sido muy poco estudiado en el país, por lo que se conoce poco de la ubicación de éstas (Tejedor et al., 2005a). De este modo, los registros de la colección podrían ayudar a determinar potenciales cuevas calientes en el país, y así empezar su estudio e inventario.

También, en la colección se destaca la presencia de Noctilio leporinus, el cual posee el mayor tamaño entre los murciélagos de la isla y es la única especie piscívora descrita (aunque también se puede alimentar de insectos). Se refugia principalmente en árboles, aunque pudiera llegar a utilizar cuevas marinas (Silva, 1979).

Por otra parte, la escasez de algunas especies pudiera explicarse por su conducta y hábitos. En el caso de L. minor, puede deberse a que suele vivir en árboles (Silva, 1979), lo cual hace más difícil su captura en las redes que usualmente se colocan en las entradas de las cuevas. La escasez de especímenes de $C$. micropus y $N$. major, reportada también en otras colecciones, puede atribuirse a que estas especies se reúnen en grupos pequeños, manteniendo bastante espacio entre individuos y estando alerta todo el tiempo (Silva, 1979 y Tejedor et al., 2005b).

Método de preservación. La mayor parte de los especímenes inventariados se encontraron preservados en alcohol. Este conservante es el preferido actualmente por los museos, ya que permite el uso de muestras para estudios genéticos, así como una mejor manipulación del espécimen para su estudio (Samarra y Carol, 1986). Además, permite la conservación de 
eventuales parásitos en el cuerpo de los individuos al ser colectados. De este modo, una buena parte de la colección de quirópteros del museo pudiera ser utilizada para futuros estudios de diversa índole.

Actualización Taxonómica. Los especímenes resultaron estar identificados por diferentes colectores, lo que pudo presentar diferencias en cuanto a las guías de identificación de especies seguidas por cada uno de ellos. Esta es la razón por la cual se encuentran registrados en la colección siete nombres a nivel sub-específico, 27 a nivel específico y 16 hasta género (Tabla 1). Muchos de estos nombres han sido utilizados en varias publicaciones en las que se incluyen murciélagos de la isla (Klingener et al., 1978; Rodríguez-Durán y Kunz, 2001; Simmons 2005; Timm y Genoways, 2003, entre otros), mostrando su validez en una época determinada. En la actualidad, la tendencia dominante es a utilizar la identificación hasta especie (Baker, 1984 y Silva, 1979), dejando a un lado, en muchos casos, las sub-especies (Simmons, 2005).

Análisis geográfico de los sitios de colecta. La mayor parte de los sitios de colecta se ubican en las regiones Sur y Este de la República Dominicana, estando principalmente representadas las provincias de Pedernales y Barahona, seguidas de San Cristóbal y San Pedro de Macorís (fig. 3). En general, esto puede explicarse por la presencia de cuevas importantes y conocidas por investigadores desde hace muchos años, tales como La Cueva de Trunicolás, Marazate y Mencía, en Pedernales; Los Patos, en Barahona, el sistema de las cuevas de El Pomier o Borbón, en San Cristóbal y La Cueva de Las Maravillas, en San Pedro de Macorís, entre otras. En la distribución de registros de acuerdo a sus colectores (fig. 4), se aprecia en las colectas cierta "regionalización" por parte de algunos colectores. Mientras que F. H. Armstrong se concentró en la Región Norte, J. A. Ottenwalder (destacándose por haber realizado el mayor número de colectas) y D.W. Stone trabajaron prioritariamente en la Región Sur-suroeste. Esto puede deberse (con la excepción de J.A. Ottenwalder) a que la mayoría de estos colectores eran extranjeros y permanecían en el país por períodos cortos de tiempo, por lo cual sólo cubrían una zona en particular o venían expresamente de vuelta a sitios ya conocidos en viajes anteriores.

Tabla 1. Actualización taxonómica de la colección del MNHNSD.*

\begin{tabular}{|l|l|}
\hline Nombre de la especie & Nombre(s) anterior(es) \\
\hline Molossus molossus (Pallas, 1766) & $\begin{array}{l}\text { Molossus major verrili } \\
\text { Molossus } \text { sp. }\end{array}$ \\
\hline Nyctinomops macrotis (Gray, 1840) & Tadarida macrotis \\
\hline Tadarida brasiliensis (I. Geoffroy, 1824) & $\begin{array}{l}\text { Tadarida brasiliensis constanzae } \\
\text { Tadarida } \text { sp. }\end{array}$ \\
\hline Mormoops blainvillei Leach, 1821 & $\begin{array}{l}\text { Mormoops blainvilli } \\
\text { Mormoops blainvilli cuvieri }\end{array}$ \\
\hline Pteronotus parnellii (Gray, 1843) & $\begin{array}{l}\text { Chyllonycteris parnelli } \\
\text { Pteronotus } \text { sp. }\end{array}$ \\
\hline Pteronotus quadridens (Gundlach, 1840) & $\begin{array}{l}\text { Pteronotus fuliginosa } \\
\text { Pteronotus } \text { sp. }\end{array}$ \\
\hline Chilonatalus micropus (Dobson, 1880) & Chilonatalus sp. \\
\hline Natalus major Miller, 1902 & \begin{tabular}{l} 
Natalus sp. \\
\hline
\end{tabular} \\
\hline
\end{tabular}

*Fuentes de sinonimia consultadas: Murray, 2008; Pierson y Rainey, 1998 y Simmons, 2005. 
Tabla 1 (continuación).

\begin{tabular}{|c|c|}
\hline Nombre de la especie & Nombre(s) anterior(es) \\
\hline Noctilio leporinus (Linnaeus, 1758) & $\begin{array}{l}\text { Noctilio leporinus mastivus } \\
\text { Noctilio sp. }\end{array}$ \\
\hline Artibeus jamaicensis Leach, 1821 & Artibeus sp. \\
\hline Brachyphylla pumila Miller, 1918 & Brachyphylla sp. \\
\hline Erophylla bombifrons (Miller, 1899) & $\begin{array}{l}\text { Erophylla bombifrons santacristobalensis } \\
\text { Erophylla sezekorni } \\
\text { Erophylla } \mathrm{sp} . \\
\text { Phyllonycteris } \mathrm{sp} .\end{array}$ \\
\hline Macrotus waterhousii Gray, 1843 & Macrotus sp. \\
\hline Monophyllus redmani Leach, 1821 & $\begin{array}{l}\text { Monophyllus cubanus ferreus } \\
\text { Monophyllus sp. } \\
\text { Phyllonycteris sp. }\end{array}$ \\
\hline Phyllonycteris poeyi Gundlach, 1861 & $\begin{array}{l}\text { Phyllonycteris obtusa } \\
\text { Phyllonycteris } \mathrm{sp} .\end{array}$ \\
\hline Phyllops falcatus (Gray, 1839) & $\begin{array}{l}\text { Phyllops haitiensis } \\
\text { Phyllops sp. }\end{array}$ \\
\hline Eptesicus fuscus (Beauvois, 1796) & Eptesicus fuscus hispaniolae \\
\hline Lasiurus minor Miller, 1931 & Lasiurus borealis \\
\hline
\end{tabular}

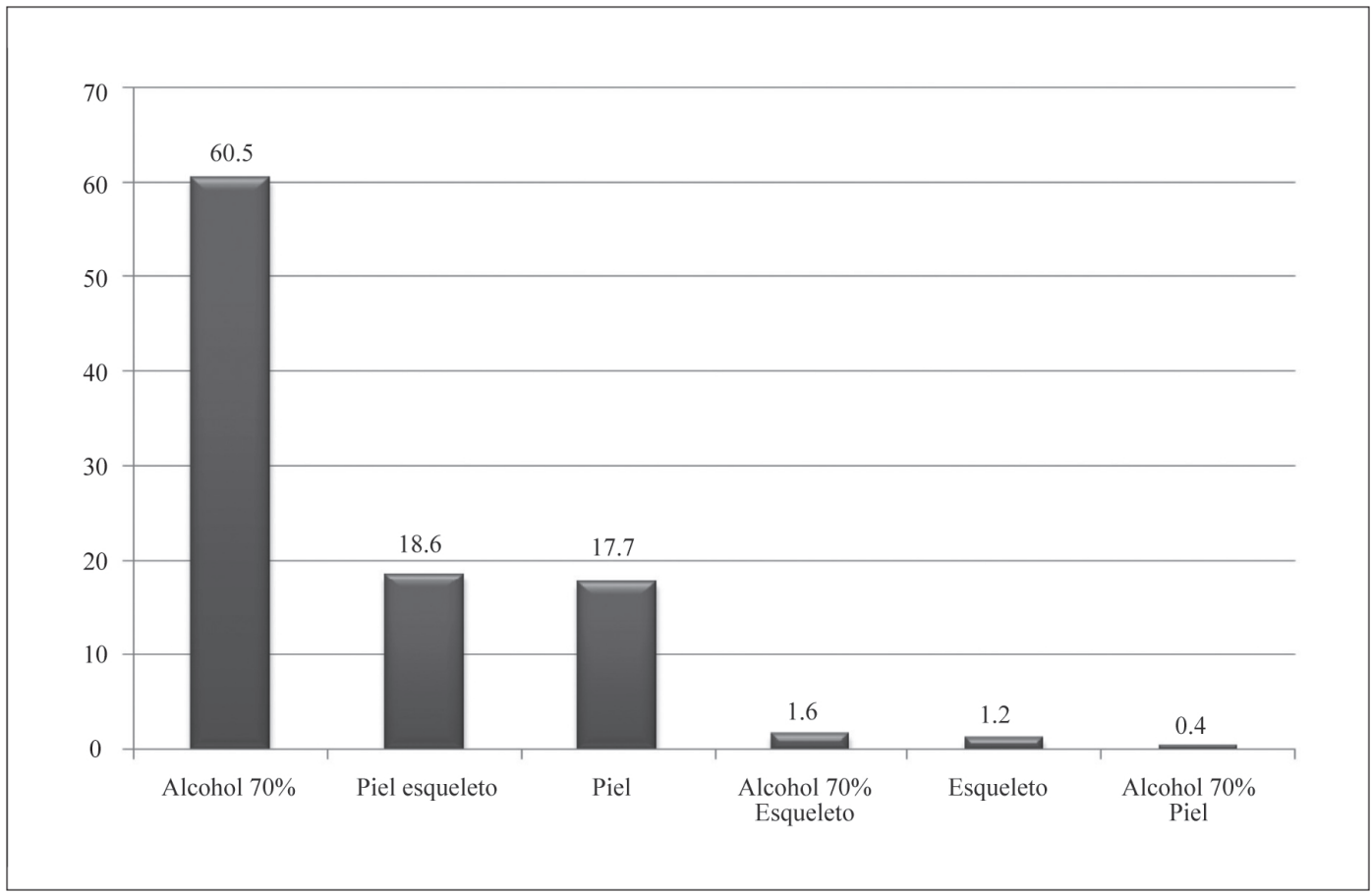

Figura 1. Porcentajes de los diferentes tipos de preservación en la colección de murciélagos del MNHNSD. 


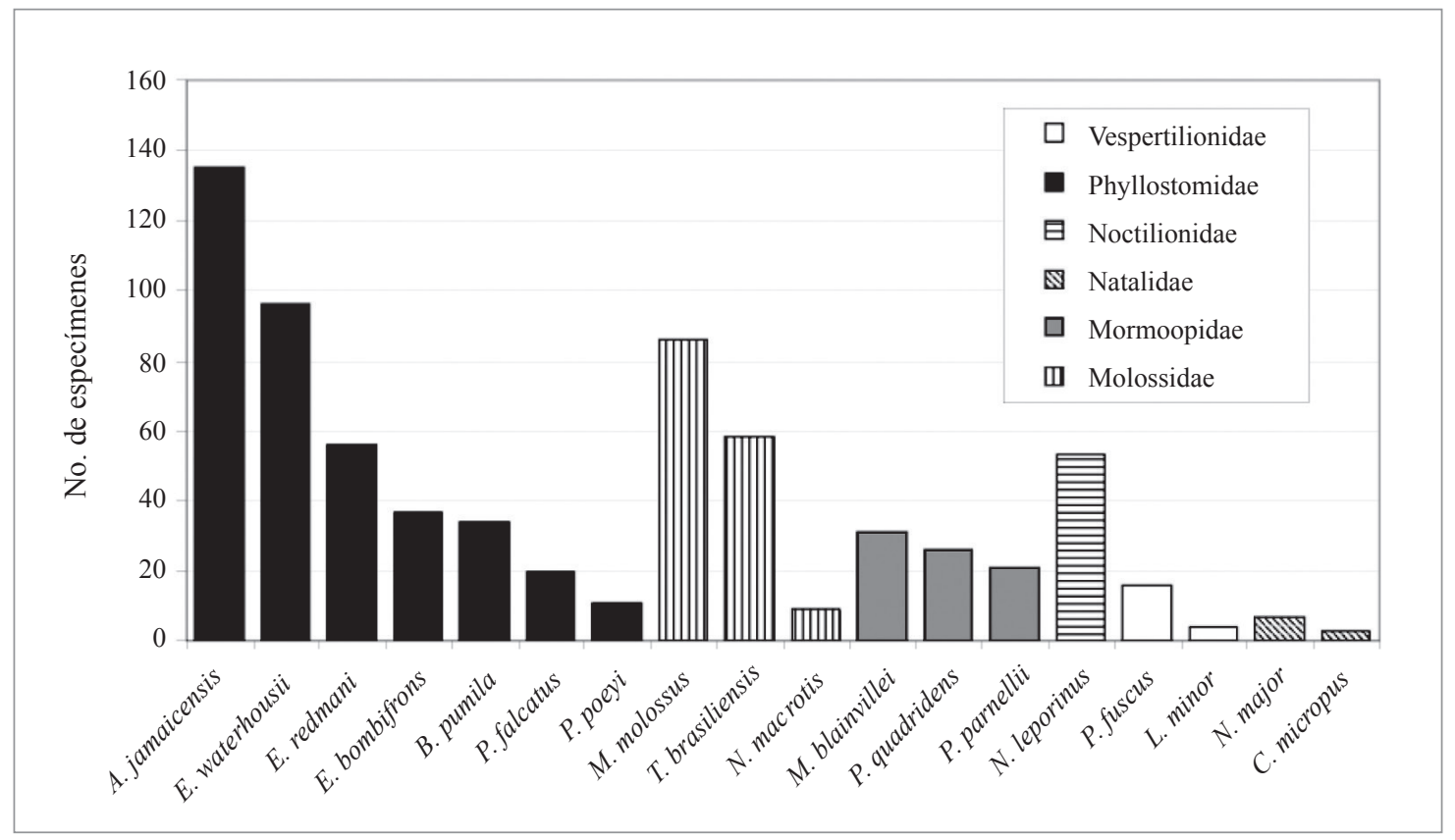

Figura 2. Distribución de los especímenes por familia y especie.

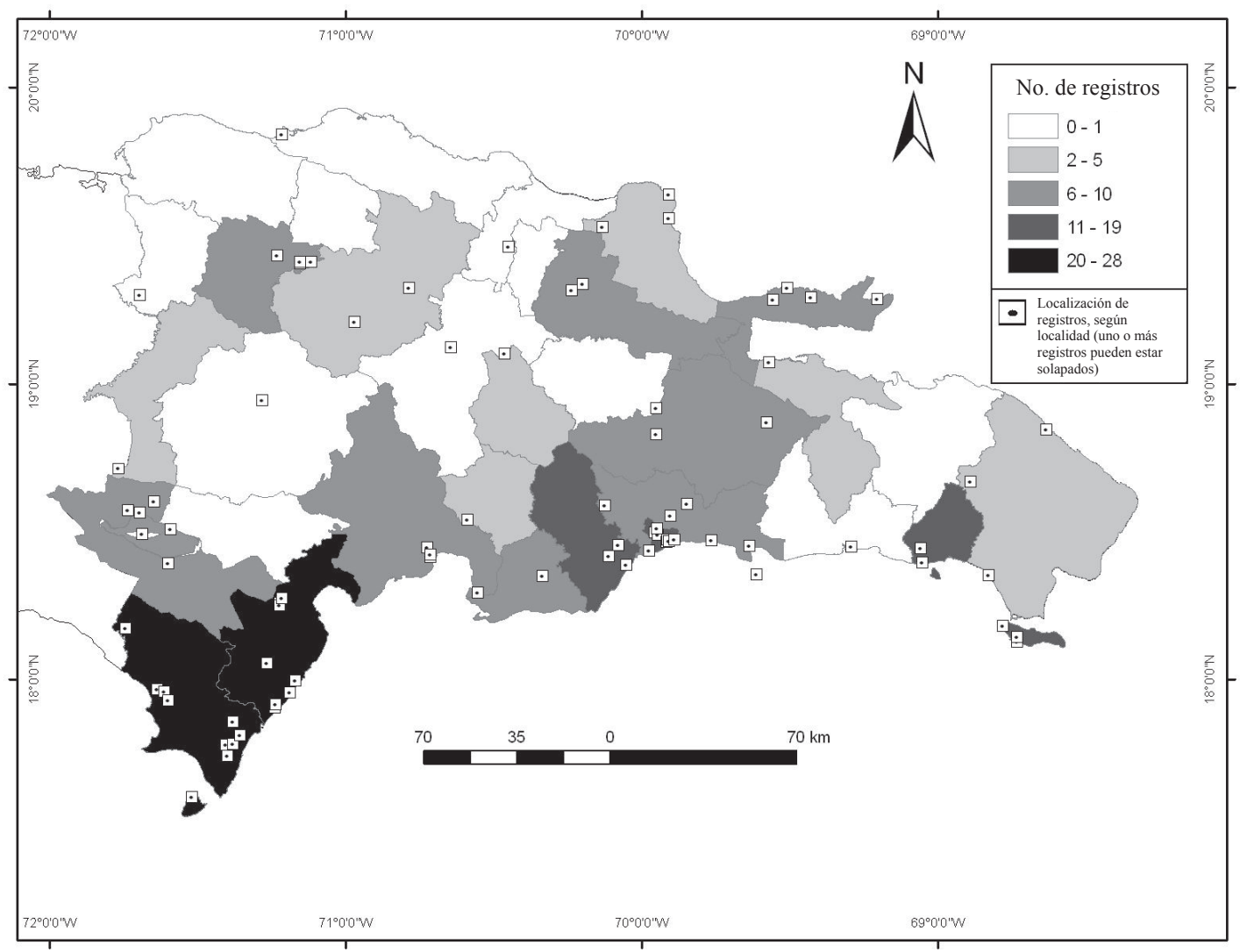

Figura 3. Distribución geográfica de los registros de la colección de murciélagos del MNHNSD. 


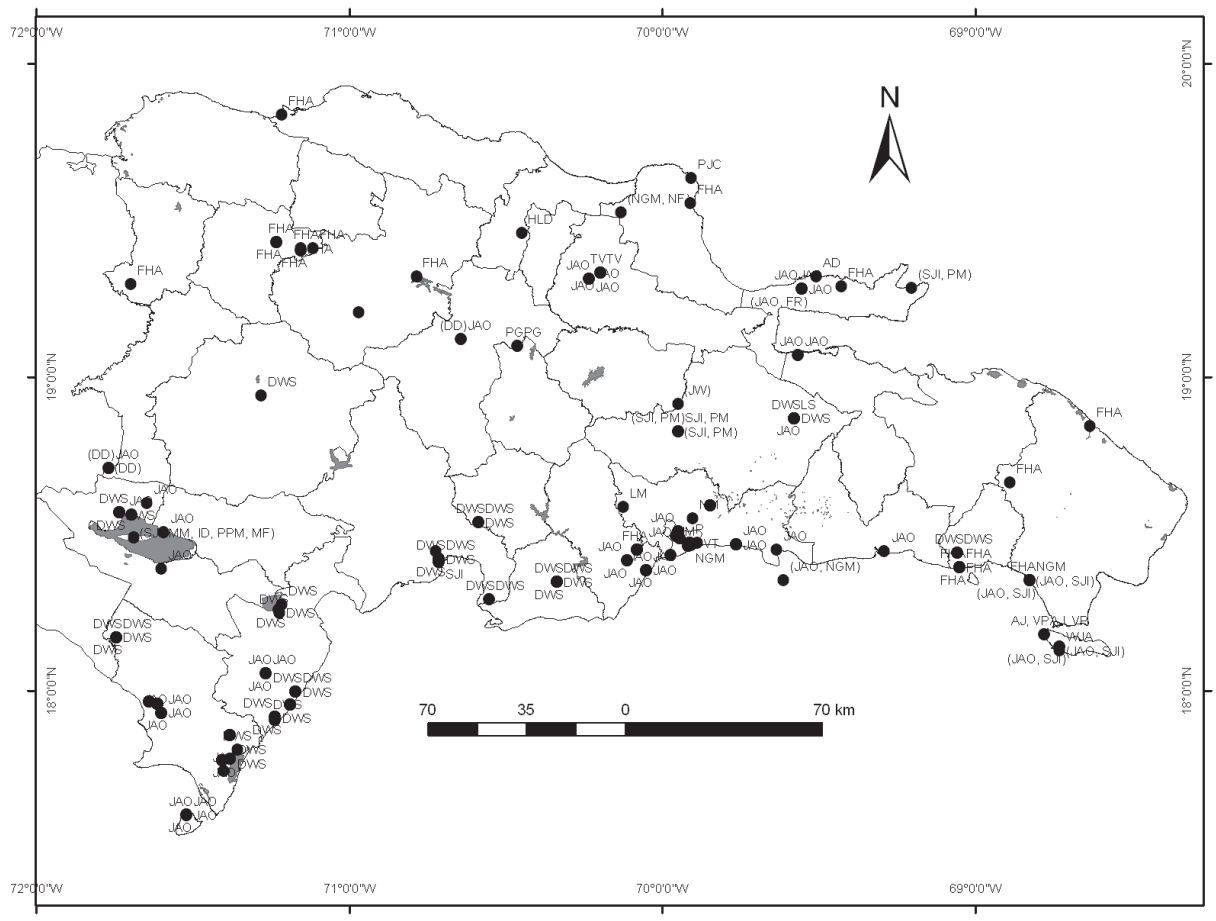

Figura 4. Localidades de colecta y su colector.

$\mathrm{AD}=$ A. Dod; AJ = A. Jiménez; DD = D. Dod; DWS=D.W. Stone; FA= F. Aznar; FHA = F. H. Armstrong; HD = H. Domínguez; ID = I. Domínguez; JAO = J.A. Ottenwalder; JW = J. Wiley; KG = K. Guerrero; LDA = L.D. Abrew; LS = L. Salazar; MF = M. Félix; MM $=$ M. Mota; NG = N. García; NF = N. Freites; SJI = S. J. Inchástegui; PJC = P.J. Cicero; P = Pilin; PG = P. Galvis; PM = P. Merejo; $\mathrm{TM}=\mathrm{T}$. Vargas; $\mathrm{VP}=\mathrm{V}$. Pérez; $\mathrm{VT}=\mathrm{V}$. Tejada; $\mathrm{WA}=\mathrm{W}$. Arendt.

\section{AGRADECIMIENTOS}

Al Museo Nacional de Historia Natural de Santo Domingo (MNHNSD), en las personas de Celeste Mir (Directora) y Carlos Suriel (Encargado Depto. de Investigación y Conservación) por las facilidades brindadas. A Cristian Marte, Elvi de los Santos, Gabriel de los Santos, Hodali Almonte, Robert Ortiz, Solanlly Carrero y Peter Sánchez, del Dpto. de Investigación y Conservación, así como a Candy Ramírez, Evelin Gabot y Kenia Ng por su valioso apoyo en los trabajos curatoriales de la colección. También a Robert J. Baker por facilitarme la clave de identificación de murciélagos de las Antillas. A Liliana Dávalos, Amy Russell y Winston Lancaster, por todo el apoyo brindado.

\section{LITERATURA CITADA}

Allen, J. A. 1908. Bats from the island of Santo Domingo. Bulletin American Museum of Natural History, 24: 580-582.

Anderson, S. 1969. Macrotus waterhousii. American Society of Mammalogists, Mammalian Species, 1: 14.

Armstrong, F. H. y M. L. Johnson. 1969. Noctilio leporinus in Hispaniola. Journal of Mammalogy, 50: 133 .

Baker, R. J. y H. H. Genoways, 1978. Zoogeography of Antillean bats. Academy of Natural Sciences of Philadelphia, Special Publication, 13: 57-97. 
Baker, R. J., J. A. Groen, y R. D. Owen. 1984. Field Key to Antillean bats. Occasional Papers, The Museum, Texas Tech University, 94: 1-18.

Baker, R. J., P. V. August y A. A. Steuer. 1978. Erophylla sezekorni. American Society of Mammalogists, Mammalian Species, 115: 1-5.

Dávalos, L. M. 2005. Molecular phylogeny of funnel-eared bats (Chiroptera: Natalidae), with notes on biogeography and conservation. Molecular Phylogenetics and Evolution, 37: 91-103.

Elliot, D. G. 1905. Descriptions of apparently new species and subspecies of mammals from Mexico and Santo Domingo. Proceedings of the Biological Society of Washington, 18: 233-236.

Fleming, T. H. 1982. Parallel trends in the species diversity of West Indian birds and bats. Oecologia, 53: 56-60.

Griffiths T. A. y D. Klingener. 1988. On the distribution of Greater Antillean bats. Biotropica, 20: $240-251$.

Herd, R. M. 1983. Pteronotus parnellii. American Society of Mammalogists, Mammalian Species, 209: 1-5.

Homan, J. A. y J. K. Jr. Jones, 1975. Monophyllus redmani. American Society of Mammalogists, Mammalian Species, 57: 1-3.

Hood, C. S. y J. K. Jr. Jones. 1984. Noctilio leporinus. American Society of Mammalogists, Mammalian Species, 216: 1-7.

Hoyt, R. A. y R. J. Baker. 1980. Natalus major. American Society of Mammalogists, Mammalian Species, 130: 1-3.

Klingener, D., H. H. Genoways y R. J. Baker. 1978. Bats from Southern Haiti. Annals of Carnegie Museum, 47 (5): 81-99.

Koopman, K .F. 1989. A review and analysis of the bats of the West Indies. Pp. 635-644, En Biogeography of the West Indies (Woods, C. A. ed.), Sandhill Crane Press, Gainesville, Florida.

Kurta, A. y R. H. Baker. 1990. Eptesicus fuscus. American Society of Mammalogists, Mammalian Species, 356: 1-10.

Lancaster, W. C. y E. K. V. Kalko. 1996. Mormoops blainvillii.American Society of Mammalogists, Mammalian Species, 544: 1-5.

McFarlane, D. A. 1989. Patterns of species co-occurrence in the Antillean bat fauna. Mammalia, 53: 59-60.

McFarlane, D. A. 1991. The species-genus relationship in Antillean bat communities. Mammalia, 55: 363-369.

McFarlane, D. A., A. Vale, K. Christenson, J. Lundberg, G. Atilles, y S. E. Lauritzen. 2000. New specimens of Late Quaternary extinct mammals from caves in Sanchez Ramirez Province, Dominican Republic. Caribbean Journal of Science, 36: 163-166. 
Miller, G. S., 1918. Three new bats from Haiti and Santo Domingo. Proceedings of the Biological Society of Washington, 31: 39-40.

Miller, B. W. y M. Miller. 2002. Results of a preliminary survey of the bats of the MayflowerBocawina National Park. Report Wildlife Conservation Society, Belize.

Milner, J., C. Jones y J. K. Jr. Jones. 1990. Nyctinomops macrotis. American Society of Mammalogists, Mammalian Species, 351: 1-4.

Murray, K. L. 2008. The genetic structure and mating system of the buffy flower bat (Erophylla sezekorni). Disertación para optar por el título de Doctor en Filosofía - Universidad de Miami.

ONE. 2006. División Territorial de la República Dominicana. Oficina Nacional de Estadística, $331 \mathrm{pp}$.

Ortega, J. y I. Castro-Arellano. 2001. Artibeus jamaicensis. American Society of Mammalogists, Mammalian Species, 662: 1-9.

Ottenwalder, J. A. 1978. Noctilio leporinus en la Isla Beata. Naturalista Postal, 32 / 78: 1.

Ottenwalder, J. A. 1979. Murciélagos del Lago Enriquillo. Naturalista Postal, 25 / 79: 1.

Ottenwalder, J. A. 1981. Murciélagos de la Isla Beata. Contribuciones Ocasionales, Museo Nacional de Historia Natural de Santo Domingo, 1: 1-7.

Pierson, E. D. y W. E. Rainey. 1998. Distribution, habitat associations, status and survey methodologies for three molossid bat species (Eumops perotis, Nyctinomops femorosaccus, Nyctinomops macrotis) and the vespertilionid (Euderma maculatum). California Department of Fish and Game. Bird and Mammal Conservation Program.

Rodríguez-Durán, A. 1998. Nonrandom aggregations of cave-dwelling bats in Puerto Rico. Journal Mammalogy, 79 (1): 141-146.

Rodríguez Duran, A., A. R. Lewis y Y. Montes. 1993. Skull morphology and diet of Antillean bat species. Caribbean Journal of Science, 29: 258-261.

Rodríguez-Durán, A. y T. H. Kunz. 1992. Pteronotus quadridens. American Society of Mammalogists, Mammalian Species, 395: 1-4.

Rodríguez-Durán, A. y T. H. Kunz. 2001. Biogeography of West Indian Bats: An ecological perspective. Pp. 355-368 En: Woods, C. H. y F. E. Sergile, (eds.) Biogeography of the West Indies. Patterns and Perspectives. CRC Press, Boca Ratón, Florida.

Samarra F. X. y A. Carol. 1986. Murciélagos incorporados a la colección del Museo de Zoología de Barcelona durante las tres últimas décadas. Miscellània Zoològica, 10: 305-314.

Sampedro-Marín A. C., C. M. Martínez-Bravo, Y. L. Otero-Fuentes, L. M. Santos-Espinosa, S. Osorio-Ozuna y A. M. Mercado-Ricardo. 2008. Presencia del murciélago casero (Molossus molossus Pallas, 1776) en la ciudad de Sincelejo, departamento de Sucre, Colombia. Caldasia, 30 (2): 495-503.

Shump, K. A. Jr. y A. U. Shump. 1982. Lasiurus borealis. American Society of Mammalogists, Mammalian Species, 183: 1-6. 
Silva Taboada, G. 1977. Algunos aspectos de la selección de hábitat en el murciélago Phyllonycteris poeyi Gundlach en Peters, 1861 (Mammalia: Chiroptera). Poeyana, 168: 1-10.

Silva Taboada, G. 1979. Los murciélagos de Cuba. Editorial Academia, La Habana, Cuba.

Simmons, N. B. 2005. Order Chiroptera. In: D. E. Wilson \& D. M. Reeder (eds.), Mammal species of the World: a taxonomic and geographic reference. Third Edition, Smithsonian Institution Press.

Swanepoel, P. y H. H. Genoways. 1983. Brachyphylla nana. American Society of Mammalogists, Mammalian Species, 206: 1-3.

Tejedor, A., V. d. C. Tavares y D. Rodríguez-Hernández. 2005a. New records of Hot-Cave Bats from Cuba and the Dominican Republic. Boletín de la Sociedad Venezolana de Espeleología, 39: $10-15$.

Tejedor, A., V. d. C. Tavares y G. Silva Taboada. 2005b. Taxonomic revision of Greater Antillean bats of the genus Natalus. American Museum Novitates, 3493: 1-22.

Timm, R. M. y H. H. Genoways. 2003. West Indian mammals from the Albert Schwartz Collection: biological and historical information. Scientific Papers, Natural History Museum, University of Kansas, 29: 1-47.

Varona, L. S. 1974. Catálogo de los mamíferos vivientes y extinguidos de las Antillas. Editorial de la Academia de Ciencias de Cuba, La Habana.

Wilkins, K. T. 1989. Tadarida brasiliensis. American Society of Mammalogists, Mammalian Species, 331: 1-10.

Wilson, D. E. y D. M. Reeder (editors). 2005. Mammal Species of the World. A Taxonomic and Geographic Reference (3rd ed), Johns Hopkins University Press, 2142 pp. Disponible en línea en: (http://www.bucknell.edu/msw3/ ). Fecha de consulta: 10 de octubre 2009. 